\title{
The Effect of Impurities on Grain Boundary Cohesion in Iron
}

\author{
J.S. Braithwaite and P. Rez
}

Department of Physics and Astronomy, Arizona State University, Tempe, AZ 82787

Impurity segregation to grain boundaries can dramatically change mechanical properties of a material. In $\mathrm{Ni}_{3} \mathrm{Al}$ boron segregation leads to cohesive enhancement, while in copper segregation of Bi to boundaries results in a dramatic embrittlement of the material. In structural steels carbon leads to greater strength while sulphur and phosphorous lower cohesive energies and make for inferior material properties. Electron microscopy has been extensively used to investigate the atomic level mechanisms responsible for these changed properties. High resolution microscopy (phase contrast or annular dark field) has been used to determine the atomic structure of well characterized boundaries and electron energy loss spectroscopy has given valuable information on changes in bonding at interfaces. To provide a framework to interpret these results theory is necessary to relate these microscopic observations to macroscopic properties. Calculations based on density functional theory can simultaneously predict atomic structure and the projected unoccupied states that are observed by EELS, as well as give estimates of the boundary cohesive energies indicating whether fracture is likely to occur [1].

We present a new survey of substitutional and interstitial impurities at the [210] tilt grain boundary in bec iron, including boron, carbon, phosphorus, and sulphur. We employ standard density functional theory calculations using the VASP [2] code, with the local density approximation for the electronic exchange-correlation, a planewave basis-set for the valence electrons, and a ultrasoft pseudopotential representation of the core electrons and nuclei. Unlike previous studies of impurities at metal grain boundaries, we take account of the relative shift of one grain against another parallel to the GB, as well as the separation perpendicular to the boundary. We include full relaxation of the atoms at the grain boundary while constraining the Fe atoms in the middle of each grain to maintain the local structure expected in bulk bcc iron.

Our simulations indicate that different grain boundary structures are adopted for interstitial (Fig. 1A) and substitutional (Fig. 1B) impurities, and that relaxation perpendicular to the GB is also highly significant. We note that the most stable geometry adopted by the GB without impurities is obtained when one grain is shifted with respect to the other parallel to the GB. Cohesive energies of the substitutional impurities studied are detailed in Table 1, where carbon and boron clearly act to stabilize the grain boundary, while phosphorus and sulphur are shown to be less stable than Fe alone. We calculate the local DOS for selected Fe atoms in our simulation cell (Fig. 1C), which can be compared directly to $\mathrm{Fe} \mathrm{L}_{23}$ spectra. Initial results indicate that changes to the electronic structure due to the presence of an impurity atom are very short ranged - Fe at a distance over $3.0 \AA$ from the impurity are identical to the bulk [3].

\section{References}

[1] P. Rez, J.A. Alvarez, Acta mater. 47 (1999) 4069.

[2] G. Kresse and J. Furthmuller Phys. Rev. B 54, (1996) 11169.

[3] This research was supported by NSF grant DMR 99-72670. 
TABLE 1. Cohesive energies, $\mathrm{E}_{\mathrm{coh}}=\mathrm{E}_{\mathrm{GB}}(\mathrm{sub})-\left[\mathrm{E}_{\mathrm{GB}}(\mathrm{vac})+\mathrm{E}_{\text {atom }}(\mathrm{sub})\right]$, and cohesive energy differences, $\Delta \mathrm{E}_{\mathrm{coh}}=\mathrm{E}_{\mathrm{coh}}(\mathrm{sub})-\mathrm{E}_{\mathrm{coh}}(\mathrm{Fe})$, for a number of substitutional impurities.

\begin{tabular}{ccc}
\hline Impurity & $\mathrm{E}_{\text {coh }}(\mathrm{eV})$ & $\Delta \mathrm{E}_{\text {coh }}(\mathrm{eV})$ \\
\hline $\mathrm{B}$ & -17.15 & -0.48 \\
$\mathrm{C}$ & -19.86 & -3.19 \\
$\mathrm{P}$ & -15.03 & 1.64 \\
$\mathrm{~S}$ & -12.04 & 4.63 \\
$\mathrm{Fe}$ & -16.67 & na \\
\hline
\end{tabular}

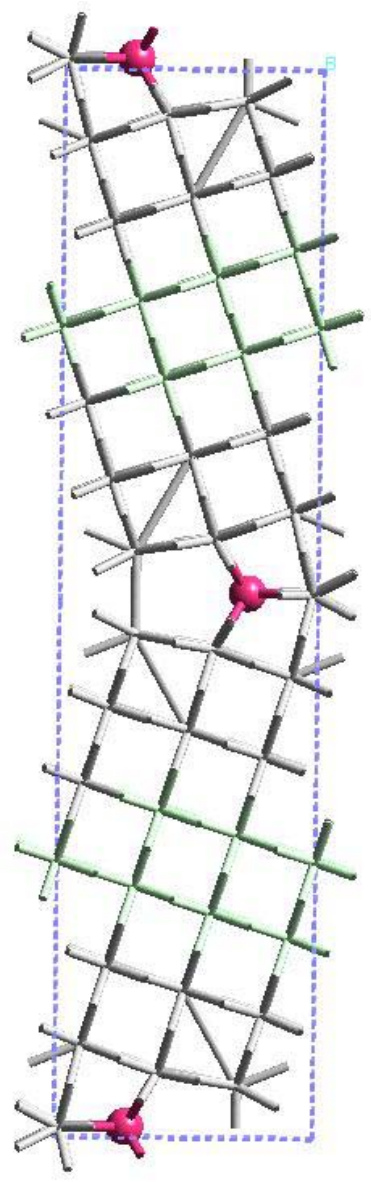

A)

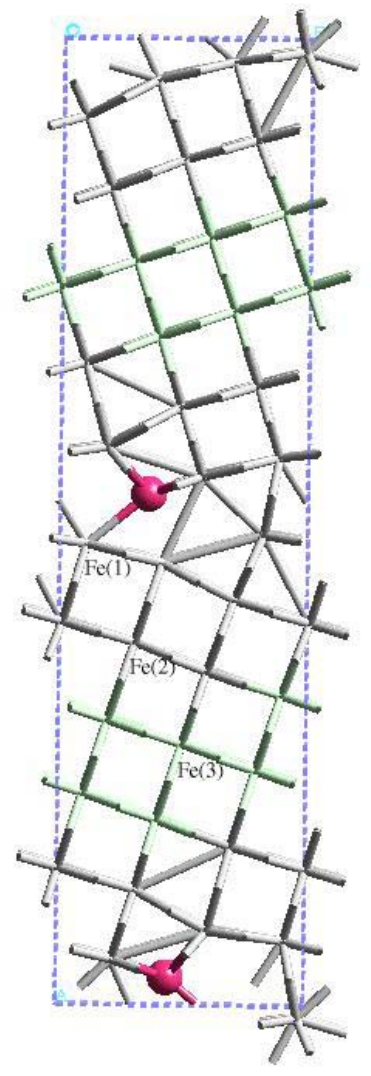

B)

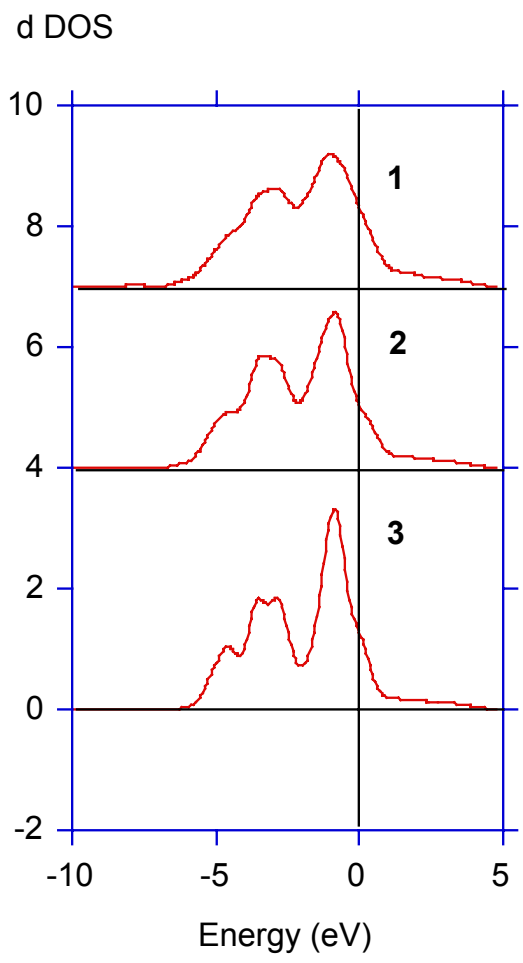

C)

FIG. 1 A) Relaxed GB with a boron interstitial. B) Relaxed GB with boron substitutional. C) Local d DOS for Fe atoms at increasing distance from the GB - refer to atom labels in B. 